\title{
State Estimation for Sampled-Data Descriptor Nonlinear System: A Strong Tracking Unscented Kalman Filter Approach
}

\author{
Tiantian Liang, ${ }^{1}$ Mao Wang, ${ }^{1}$ and Zhenhua Zhou ${ }^{2}$ \\ ${ }^{1}$ School of Astronautics, Harbin Institute of Technology, West Dazhi Street, Harbin 150001, China \\ ${ }^{2}$ Changzhou Vocational Institute of Light Industry, Changzhou, China \\ Correspondence should be addressed to Tiantian Liang; liangtiantian1122@163.com
}

Received 15 January 2017; Revised 15 May 2017; Accepted 15 June 2017; Published 8 August 2017

Academic Editor: Mohammad D. Aliyu

Copyright (c) 2017 Tiantian Liang et al. This is an open access article distributed under the Creative Commons Attribution License, which permits unrestricted use, distribution, and reproduction in any medium, provided the original work is properly cited.

\begin{abstract}
This paper proposes a state estimation method for a sampled-data descriptor system by the Kalman filtering method. The sampleddata descriptor system is firstly discretized to obtain a discrete-time nonsingular model. Based on the discretized nonsingular system, a strong tracking unscented Kalman filter (STUKF) algorithm is designed for the state estimation. Then, a defined suboptimal fading factor is proposed and added to the prediction covariance for decreasing the weight of the prior knowledge on the conventional UKF filtering solution. Finally, a simulation example is given to show the effectiveness of the proposed method.
\end{abstract}

\section{Introduction}

Descriptor systems, which can be also defined as singular systems or differential-algebraic equation systems, were proposed by the famous scholar H. H. Rosenbrock in 1970s [1]. For some practical systems, descriptor system theory has a better characterization than the space description method, such as in electrical networks [2], constrained mechanical systems [3], and aircraft modeling [4]. For this reason, the descriptor system has received more and more attention in the last decade. Especially in the domain of state estimation for the descriptor systems, prominent methods such as the design of the descriptor observer have been proposed by the former studies [5-9]. But, for most of the practical systems, there exist noises in both state vector and measurement which makes the state estimation problem more complex. It is well known that the Kalman filter method can solve this problem efficiently. So, this work is based on this background to study the Kalman filter design for a precise state estimation of the descriptor systems with noise and guarantee the convergence of the estimate.

Some preliminary works have been established for the effective research of the Kalman filter for descriptor systems [10-14]. In [15], a "Hamiltonian" pencil is proposed for solving the optimal control problem of the descriptor system, where the covariance of the measurement noise may be singular. For the models of time-varying descriptor system, a "dual approach" is proposed in [16] to deduce a "3-block" form for the optimal filter and a corresponding 3-block Riccati equation. A further research for the state estimation of general discrete-time linear descriptor systems based on a recursive restructuring algorithm is proposed for the models in [17] by transforming the estimation problem into the one which future dynamics do not influence in the present state. However, for the descriptor systems, the optimal filter parameters of the descriptor systems in practical works may be singular ones which are hard to calculate $[16,17]$. So, there is a great need for us to develop a novel Kalman filter for the state estimation of the descriptor system.

Recently, a strong tracking unscented Kalman filter (STUKF) method was proposed in [18] to investigate state estimation for discrete-time systems. In the presence of process model uncertainty, a defined suboptimal fading factor is introduced into the prediction covariance to adjust the Kalman gain matrix online. This suboptimal fading factor is derived based on the orthogonality principle of the innovation vectors in the framework of the STUKF, which avoids the increase of estimation error caused by the uncertainties in the system. 
Based on the results in [18], a new approach of state estimation for sampled-data descriptor systems is proposed in this paper. The proposed sampled-data descriptor system is firstly discretized to obtain a discrete-time nonsingular model. As parameters are introduced for transforming the descriptor model into a nonsingular one, this transforming way leads to an increase in the dynamic model of the nonsingular system. As a result, there is an increase in the corresponding covariance matrix, so the standard UKF cannot calculate the corresponding covariance matrix of the transformed nonsingular system accurately.

To solve this problem, a strong tracking unscented Kalman filter (STUKF) algorithm which has strong robustness against the increasing noise in the dynamic model of the transformed nonsingular system is proposed in this paper. A defined suboptimal fading factor based on the orthogonality principle of the innovation vectors in STUKF is added to the prediction covariance for decreasing the weight of the prior knowledge on the filtering solution and adjusting the Kalman gain matrix of the transformed nonsingular system. By this suboptimal fading factor, the proposed STUKF can effectively decrease the influence of the increasing noise in the transformed nonsingular system, which means the proposed STUKF can guarantee the accuracy of the state estimation for the sampled-data descriptor nonlinear system.

This paper is organized as follows. In Section 2, a model of the sampled-data descriptor system is proposed, and then parameters are given to discretize and transform the proposed sampled-data system into a discrete-time nonsingular one. Two assumptions are given for the further research of the Kalman filter for the proposed sampled-data descriptor system. In Section 3, an unscented Kalman filter is proposed for a normal system. The process of the UKF algorithm is given for the normal system. In Section 4, based on the UKF algorithm in Section 3, a strong tracking unscented Kalman filter (STUKF) is proposed for state estimation and implemented in the transformed nonsingular system. In Section 5, a simulation example is given to demonstrate the validity of our results. Finally, conclusions are drawn in Section 6 .

\section{Problem Statement and Assumptions}

Consider the following sampled-data nonlinear descriptor system:

$$
\begin{aligned}
& E \dot{x}(t)=\varphi(x(t))+w(t), \\
& y\left(t_{k}\right)=C x\left(t_{k}\right)+v\left(t_{k}\right),
\end{aligned}
$$

where $x \in R^{n}$ is the state vector and $y\left(t_{k}\right) \in R^{m}$ is the sampled output at time instant $t_{k}$ with the sampling interval $\tau=t_{k+1}-$ $t_{k}$. In system (1), $E$ satisfies the notion that $\operatorname{rank}(E)=r \leq n$, which means that $E$ may be singular. Additionally, $\left[\begin{array}{c}E \\ C\end{array}\right]$ satisfy that $\operatorname{rank}\left[\begin{array}{c}E \\ C\end{array}\right]=n$.

For system (1), because the singular matrix $E$ exists in the dynamic model, it is very hard to calculate the covariance matrix in the process of Kalman filtering, In this paper, our purpose is to find parameters to transform the descriptor system (1) into a nonsingular one. Since $\operatorname{rank}\left[\begin{array}{l}E \\ C\end{array}\right]=n$, there exists a full-rank matrix satisfying

$$
T E+N C=I_{n+m},
$$

where $T$ and $N$ are defined as the general solution matrix for $\left[\begin{array}{ll}T & N\end{array}\right]$ given by

$$
\left[\begin{array}{ll}
T & N
\end{array}\right]=\left[\begin{array}{l}
E \\
C
\end{array}\right]^{\dagger}+S\left(I_{n+m}-\left[\begin{array}{l}
E \\
C
\end{array}\right]\left[\begin{array}{l}
E \\
C
\end{array}\right]^{\dagger}\right)
$$

But it is easy to see that the dynamic model of (1) is a continuous-time one, and the measurement is a discretetime one. So, we firstly discretize system (1) to be a discrete nonsingular model.

For any $x(t)$, the following equation exists based on (2):

$$
\begin{aligned}
\dot{x}(t) & =(T E+N C) \dot{x}(t)=T E \dot{x}(t)+N C \dot{x}(t) \\
& =T[\varphi(x(t))+w(t)]+N C \dot{x}(t) \\
& =T \varphi(x(t))+T w(t)+N C \dot{x}(t) .
\end{aligned}
$$

Then, using the Euler discretization method, (4) can be discretized as

$$
x_{k}=T x_{k-1}+T \tau \varphi\left(x_{k-1}\right)+T w_{k}+N C_{k} x_{k}+O\left(\tau^{2}\right),
$$

where

$$
\begin{aligned}
& O\left(\tau^{2}\right)=T\left[\left(\frac{\tau^{2}}{2 !}\right) \dot{\varphi}(x(t))+\left(\frac{\tau^{3}}{3 !}\right) \varphi^{(2)}(x(t))+\cdots\right. \\
& \left.+\left(\frac{\tau^{k}}{k !}\right) \varphi^{(k-1)}(x(t))\right]
\end{aligned}
$$

and $C_{k}$ is the measurement matrix $C$ at time $t_{k}$.

In this paper, the sampling interval $\tau$ is chosen to be sufficiently small so that the terms in $O\left(\tau^{2}\right)$ can be omitted.

The measurement is represented as

$$
y_{k}=C_{k} x_{k}+v_{k}
$$

So, system (1) is discretized and represented as the following equations:

$$
\begin{aligned}
& x_{k}=T x_{k-1}+T \tau \varphi\left(x_{k-1}\right)+T w_{k}+N C_{k} x_{k}, \\
& y_{k}=C_{k} x_{k}+v_{k} .
\end{aligned}
$$

Equation (7) can be rewritten as

$$
C_{k} x_{k}=y_{k}-v_{k}
$$

So, (8) can be rewritten as

$$
\begin{aligned}
& x_{k}=T x_{k-1}+T \tau \varphi\left(x_{k-1}\right)+T w_{k}+N y_{k}-N v_{k}, \\
& y_{k}=C_{k} x_{k}+v_{k} .
\end{aligned}
$$

In (10), $w_{k}$ and $v_{k}$ are separately white Gaussian noise sequence with zero mean and their covariance matrix satisfies $Q_{k}=E\left[w_{k} w_{k}^{T}\right], R_{k}=E\left[v_{k} v_{k}^{T}\right]$. 
Denote

$$
\begin{aligned}
f\left(x_{k-1}\right) & =T x_{k-1}+T \tau \varphi\left(x_{k-1}\right)+N y_{k}, \\
\Delta E_{k} & =T w_{k}-N v_{k} .
\end{aligned}
$$

Submitting (11) into (10), system (10) can be written as the following equations:

$$
\begin{aligned}
& x_{k}=f\left(x_{k-1}\right)+\Delta E_{k}, \\
& y_{k}=C_{k} x_{k}+v_{k},
\end{aligned}
$$

where $\Delta E_{k}$ is the noise in the dynamic model of the new system (12).

The following assumptions are proposed for the Kalman filter of the sampled-data nonlinear descriptor system.

Assumption 1. $C_{k}$ has full-column rank.

Assumption 2. [ [ $C_{k} R_{k}$ ] has full-row rank [15].

\section{Unscented Kalman Filter (UKF)}

Firstly, consider a normal system model as follows:

$$
\begin{aligned}
& x_{k}=f\left(x_{k-1}\right)+w_{k}, \\
& y_{k}=C_{k} x_{k}+v_{k} .
\end{aligned}
$$

For system (13), the state estimation by the standard UKF algorithm is described as follows.

Step 1. Give the state estimation $\widehat{x}_{k-1}$ and the error covariance $P_{k-1}$. The sigma points are selected by

$$
\begin{aligned}
& \xi_{i, k-1}=\widehat{x}_{k-1}, \quad i=0, \\
& \xi_{i, k-1}=\widehat{x}_{k-1}+a\left(\sqrt{n P_{k-1}}\right)_{i}, \quad i=1,2, \ldots, n, \\
& \xi_{i, k-1}=\widehat{x}_{k-1}-a\left(\sqrt{n P_{k-1}}\right)_{i},
\end{aligned}
$$

where $a \in R$ is a tuning parameter which denotes the spread of the sigma points around $\widehat{x}_{k-1}$ and is set to be sufficiently small. $a \sqrt{n P_{k-1}}$ is the $i$ th column of the matrix square root of $n P_{k-1}$.

Step 2 (prediction). Each of the sigma points is instantiated through the process model to yield a set of transformed samples:

$$
\xi_{i, k / k-1}=f\left(\xi_{i, k-1}\right)
$$

The predicted mean and prediction covariance are updated by

$$
\begin{aligned}
\widehat{x}_{k / k-1}= & \sum_{i=0}^{2 n} \omega_{i} \xi_{i, k / k-1}, \\
P_{k / k-1}= & \sum_{i=0}^{2 n} \omega_{i}\left(\xi_{i, k / k-1}-\widehat{x}_{k / k-1}\right)\left(\xi_{i, k / k-1}-\widehat{x}_{k / k-1}\right)^{T} \\
& +Q_{k},
\end{aligned}
$$

where

$$
\begin{aligned}
& \omega_{i}=1-\frac{1}{a^{2}}, \quad i=0, \\
& \omega_{i}=\frac{1}{2 \pi a^{2}}, \quad i=1,2, \ldots, 2 n .
\end{aligned}
$$

Step 3 (update). A new set of sigma points are recomputed with the mean of $\widehat{x}_{k / k-1}$ and the covariance of $P_{k / k-1}$.

The sigma points for the measurements are

$$
\gamma_{i, k / k-1}=C_{k} \xi_{i, k / k-1}, \quad i=0,1, \ldots, 2 n .
$$

The weighted mean and covariance of the predicted measurements are calculated as

$$
\begin{aligned}
\widehat{y}_{k / k-1}= & \sum_{i=0}^{2 n} \omega_{i} \gamma_{i, k / k-1}, \\
P_{\widehat{y}_{k / k-1}}= & \sum_{i=0}^{2 n} \omega_{i}\left(\gamma_{i, k / k-1}-\widehat{y}_{k / k-1}\right)\left(\gamma_{i, k / k-1}-\widehat{y}_{k / k-1}\right)^{T} \\
& +R_{k} .
\end{aligned}
$$

The cross-covariance between the predicted state and measurement is given by

$$
\begin{aligned}
& P_{\widehat{x}_{k / k-1} \widehat{y}_{k / k-1}} \\
& \quad=\sum_{i=0}^{2 n} \omega_{i}\left(\xi_{i, k / k-1}-\widehat{x}_{k / k-1}\right)\left(\gamma_{i, k / k-1}-\widehat{y}_{k / k-1}\right)^{T}+Q_{k} .
\end{aligned}
$$

The Kalman gain is determined by

$$
K_{k}=P_{\widehat{x}_{k / k-1} \widehat{y}_{k / k-1}} P^{-1} \widehat{y}_{k / k-1} .
$$

Then, the estimation $\widehat{x}_{k}$ and the corresponding error covariance matrix $P_{k}$ can be updated as

$$
\begin{aligned}
& \hat{x}_{k}=\widehat{x}_{k / k-1}+K_{k}\left(y_{k}-\widehat{y}_{k / k-1}\right), \\
& P_{k}=P_{k / k-1}-K_{k} P_{\hat{y}_{k / k-1}} K_{k}^{T} .
\end{aligned}
$$

Step 4. Steps 1 to 3 are repeated for the next time step.

In the following work, we consider the nonsingular system (12) transformed from the proposed descriptor system (1). In system (12), define

$$
\widetilde{Q}_{k}=E\left[\left(\Delta E_{k}\right)\left(\Delta E_{k}\right)^{T}\right]=T Q_{k} T^{T}+N R_{k} N^{T} .
$$


So, the prediction covariance matrix of system (12) is

$$
\begin{aligned}
\widetilde{P}_{k / k-1}= & \sum_{i=0}^{2 n} \omega_{i}\left(\xi_{i, k / k-1}-\widehat{x}_{k / k-1}\right)\left(\xi_{i, k / k-1}-\widehat{x}_{k / k-1}\right)^{T} \\
& +\widetilde{Q}_{k}
\end{aligned}
$$

which means that there is an increase in the covariance matrix of system (12) compared with the normal system (13). For eliminating the influence of this increase, we need to propose a substituted algorithm based on the standard UKF for an accurate state. Based on this idea, a strong tracking unscented Kalman filter (STUKF) is proposed for the state estimation of system (12).

\section{Strong Tracking Unscented Kalman Filter (STUKF)}

The key of the strong tracking unscented Kalman filter (STUKF) is to incorporate a time-varying suboptimal fading factor into the prediction covariance to decrease the impact of prior knowledge on the current state estimation. Based on the time-varying suboptimal fading factor, the modified prediction covariance equation is illustrated as follows:

$$
\begin{gathered}
P_{k / k-1}{ }^{*}=\lambda_{k}\left\{\sum _ { i = 0 } ^ { 2 n } \omega _ { i } \left[\left(\xi_{i, k / k-1}-\widehat{x}_{k / k-1}\right)\right.\right. \\
\left.\left.\cdot\left(\xi_{i, k / k-1}-\widehat{x}_{k / k-1}\right)^{T}+\widetilde{Q}_{k}\right]\right\} .
\end{gathered}
$$

By adjusting the time-varying suboptimal fading factor $\lambda_{k}$, the increase in the covariance matrix in (12) can effectively be decreased.

In the following work, the deducing of the time-varying suboptimal factor is given.

Denote the innovation vector by

$$
\tilde{y}_{k}=y_{k}-\widehat{y}_{k / k-1} \text {. }
$$

The aforementioned suboptimal fading factor can be determined by solving the following equations:

$$
\begin{aligned}
E\left[\left(x_{k}-\hat{x}_{k}\right)\left(x_{k}-\hat{x}_{k}\right)^{T}\right] & =\min , \\
E\left[\tilde{y}_{k}^{T} \cdot \tilde{y}_{k+j}\right] & =0, \quad j=1,2, \ldots
\end{aligned}
$$

The first equation of (28) is the criteria for a filter to reach the optimal solution. The second equation of (28) is called the orthogonality principle. This principle requires the innovation sequences to be orthogonal to each other at each time instant to show that all the useful information in the innovation sequence is extracted $[19,20]$.

The estimation error and prediction error are defined as

$$
\begin{aligned}
\tilde{x}_{k} & =x_{k}-\widehat{x}_{k}, \\
\tilde{x}_{k / k-1} & =x_{k}-\widehat{x}_{k / k-1} .
\end{aligned}
$$

The weighted mean is defined as

$$
\widehat{y}_{k / k-1}=C_{k} \widehat{x}_{k / k-1} \text {. }
$$

An unknown matrix $\beta_{k}=\operatorname{diag}\left(\beta_{1, k}, \beta_{2, k}, \ldots, \beta_{n, k}\right)$ is introduced into the predicted mean the following equation is obtained:

$$
\tilde{x}_{k / k-1}=\beta_{k} F_{k} \tilde{x}_{k-1}+\Delta E_{k}
$$

where

$$
F_{k}=\frac{\partial(f(x))}{\partial x} \mid x=\widehat{x}_{k-1} .
$$

From (12), (27), and (31), we have

$$
\begin{aligned}
\tilde{y}_{k} & =C_{k}\left(x_{k}-\widehat{x}_{k / k-1}\right)+v_{k} \\
& =C_{k}\left(\beta_{k} F_{k} \tilde{x}_{k-1}+\Delta E_{k}\right)+v_{k} .
\end{aligned}
$$

Define $y_{j, k}=E\left[\tilde{y}_{k+j} \tilde{y}_{k}^{T}\right]$, and the properties of Gaussian white noise satisfy $E\left[w_{i} w_{j}^{T}\right]=0, E\left[w_{i} v_{j}^{T}\right]=0(i \neq j)$, and $E\left[w_{i} v_{j}^{T}\right]=0$.

It is verified that

$$
\begin{aligned}
y_{j, k}= & E\left[\widetilde{y}_{k+j} \tilde{y}_{k}^{T}\right] \\
= & E\left\{C_{k+j}\left[\beta_{k+j} F_{k+j} \tilde{x}_{k+j-1}+\Delta E_{k+j}\right]+v_{k+j}\right\} \\
& \times\left\{C_{k}\left[\beta_{k} F_{k} \tilde{x}_{k-1}+\Delta E_{k}\right]+v_{k}\right\}^{T} \\
= & C_{k}\left(\prod_{i=k+1}^{k+j-1}\left(I-K_{i} C_{i}\right)\right) f_{i} \\
& \cdot\left(P_{\widehat{x}_{k / k-1} \hat{y}_{k / k-1}}-K_{k} y_{0, k}\right) .
\end{aligned}
$$

The weighted covariance of the predicted measurements is defined as

$$
\begin{aligned}
P_{\widehat{y}_{k / k-1}} & =E\left[\left(y_{k}-\widehat{y}_{k / k-1}\right)\left(y_{k}-\widehat{y}_{k / k-1}\right)^{T}\right] \\
& =C_{k} P_{k / k-1} C_{k}^{T}+R_{k} .
\end{aligned}
$$

The cross-covariance between the predicted state and measurement is defined as

$$
\begin{aligned}
P_{\widehat{x}_{k / k-1} \hat{y}_{k / k-1}} & =E\left[\left(x_{k}-\widehat{x}_{k / k-1}\right)\left(y_{k}-\widehat{y}_{k / k-1}\right)^{T}\right] \\
& =P_{k / k-1} C_{k}^{T} .
\end{aligned}
$$

In (35), let $y_{j, k}=0$; it is obtained that

$$
P_{\widehat{x}_{k / k-1} \hat{y}_{k / k-1}}-K_{k} y_{0, k}=0 .
$$

Substitute (35), (36), and (22) into (37); it is obtained that

$$
P_{k / k-1} C_{k}^{T}\left(I-\left(C_{k} P_{k / k-1} C_{k}^{T}+R_{k}\right)^{-1} y_{0, k}\right)=0,
$$

which means

$$
C_{k} P_{k / k-1} C_{k}^{T}=y_{0, k}-R_{k}
$$


Substitute (26) into (39) to take place of $P_{k / k-1}$; it is obtained that

$$
C_{k} P_{k / k-1}^{*} C_{k}^{T}=y_{0, k}-R_{k} .
$$

Equation (40) is equivalent to the following equation:

$$
\begin{aligned}
& C_{k} \lambda_{k} \sum_{i=0}^{2 n} \omega_{i}\left[\left(\xi_{i, k / k-1}-\widehat{x}_{k / k-1}\right)\left(\xi_{i, k / k-1}-\widehat{x}_{k / k-1}\right)^{T}\right] C_{k}^{T} \\
& \quad=y_{0, k}-R_{k}-C_{k} \widetilde{Q}_{k} C_{k}^{T} .
\end{aligned}
$$

In (41), $y_{0, k}$ can be calculated by the following equation:

$$
y_{0, k}= \begin{cases}\tilde{y}_{1} \tilde{y}_{1}^{T}, & k=1, \\ \frac{\rho y_{0, k-1}+\tilde{y}_{k} \tilde{y}_{k}^{T}}{1+\rho}, & k>1,\end{cases}
$$

in which $0 \leq \rho \leq 1$ is a forgetting factor and is generally set as $\rho=0.95$.

By taking the trace of both sides of (42), the suboptimal fading factor can be described as

$$
\begin{aligned}
& \lambda_{k} \\
& =\frac{\operatorname{tr}\left(y_{0, k}-R_{k}-C_{k} \widetilde{Q}_{k} C_{k}^{T}\right)}{\operatorname{tr}\left(\sum_{i=0}^{2 n} \omega_{i}\left(\gamma_{i, k / k-1}-\widehat{y}_{k / k-1}\right)\left(\gamma_{i, k / k-1}-\widehat{y}_{k / k-1}\right)^{T}\right)} .
\end{aligned}
$$

It can be seen in (43) that $\lambda_{k}$ may be less than 1 . To solve this problem, the suboptimal fading vector can be further chosen as

$$
\begin{aligned}
\lambda_{k} & =\max \{1, \\
& \left.\frac{\operatorname{tr}\left(y_{0, k}-R_{k}-C_{k} \widetilde{Q}_{k} C_{k}^{T}\right)}{\operatorname{tr}\left(\sum_{i=0}^{2 n} \omega_{i}\left(\gamma_{i, k / k-1}-\widehat{y}_{k / k-1}\right)\left(\gamma_{i, k / k-1}-\widehat{y}_{k / k-1}\right)^{T}\right)}\right\} \\
= & \max \{1, \\
& \left.\frac{\operatorname{tr}\left(y_{0, k}-R_{k}-C_{k}\left(T Q_{k} T^{T}+N R_{k} N^{T}\right) C_{k}^{T}\right)}{\operatorname{tr}\left(\sum_{i=0}^{2 n} \omega_{i}\left(\gamma_{i, k / k-1}-\widehat{y}_{k / k-1}\right)\left(\gamma_{i, k / k-1}-\widehat{y}_{k / k-1}\right)^{T}\right)}\right\} .
\end{aligned}
$$

By the standard UKF and introduced time-varying suboptimal fading factor $\lambda_{k}$, a STUKF algorithm is proposed for the transformed nonsingular nonlinear system (12). steps.

The procedure of the STUKF involves the following main

Step 1. Give the state estimate $\widehat{x}_{k / k-1}$ and the error covariance matrix $P_{k-1}$.

Step 2. Implement the standard UKF prediction procedure from (14) to (16).

Then, update the procedure as (19) to (23).
Step 3. Replace $P_{k / k-1}$ in the UKF with its modified type $P_{k / k-1}^{*}$ which is given in (26).

Step 4. Repeat Steps 1 to 3 for the next time step.

\section{Simulation}

In this section, a simulation example is given to show the effectiveness of the proposed method.

Consider the following discrete descriptor system discretized by the sampled-data descriptor system in the form of (1) with sampling interval $\tau=0.1 \mathrm{~S}$.

$$
\begin{aligned}
& E x_{k} \\
& =\left[\begin{array}{c}
x_{1, k-1}+h\left(-\mu x_{1, k-1}+\frac{K}{T_{r}} x_{3, k-1}+K p x_{5, k-1} x_{4, k-1}\right) \\
x_{2, k-1}+h\left(-\mu x_{2, k-1}-K p_{5, k-1} x_{3, k-1}+\frac{K}{T_{r}} x_{4, k-1}\right) \\
x_{3, k-1}+h\left(\frac{M}{T_{r}} x_{2, k-1}-\frac{1}{T_{r}} x_{3, k-1}-p x_{5, k-1} x_{4, k-1}\right) \\
x_{1, k-1}+h\left(\frac{M}{T_{r}} x_{2, k-1}+p x_{5, k-1} x_{3, k-1}-\frac{1}{T_{r}} x_{4, k-1}\right) \\
x_{1, k-1}+h\left(\frac{p M}{J L_{r}}\left(x_{3, k-1} x_{2, k-1}-x_{4, k-1} x_{1, k-1}\right)-\frac{T_{r}}{J}\right)
\end{array}\right],
\end{aligned}
$$

where

$$
E=\left[\begin{array}{lllll}
1 & 0 & 0 & 0 & 0 \\
0 & 1 & 0 & 0 & 0 \\
0 & 0 & 1 & 0 & 0 \\
0 & 0 & 0 & 1 & 0 \\
0 & 0 & 0 & 0 & 0
\end{array}\right],
$$$$
C_{k}=\left[\begin{array}{lllll}
1 & 0 & 0 & 0 & 0 \\
0 & 1 & 0 & 0 & 0 \\
0 & 0 & 1 & 0 & 0 \\
0 & 0 & 0 & 1 & 0 \\
0 & 0 & 0 & 0 & 1
\end{array}\right] \text {. }
$$

The initial conditions are chosen as

$$
\begin{aligned}
x_{1,0} & =x_{2,0}=x_{3,0}=x_{4,0}=x_{5,0}=0, \\
\widehat{x}_{1,0} & =200, \\
\widehat{x}_{2,0}, & =200, \\
\widehat{x}_{3,0} & =50, \\
\widehat{x}_{4,0} & =50, \\
\widehat{x}_{5,0} & =350 .
\end{aligned}
$$

The initial condition of the covariance matrix is

$$
\widehat{P}_{0}=100^{2} I_{5} \text {. }
$$

The covariance matrices of $Q_{k}$ and $R_{k}$ are

$$
\begin{aligned}
& Q_{k}=10^{-4} I_{5}, \\
& R_{k}=0.01 I_{5} .
\end{aligned}
$$




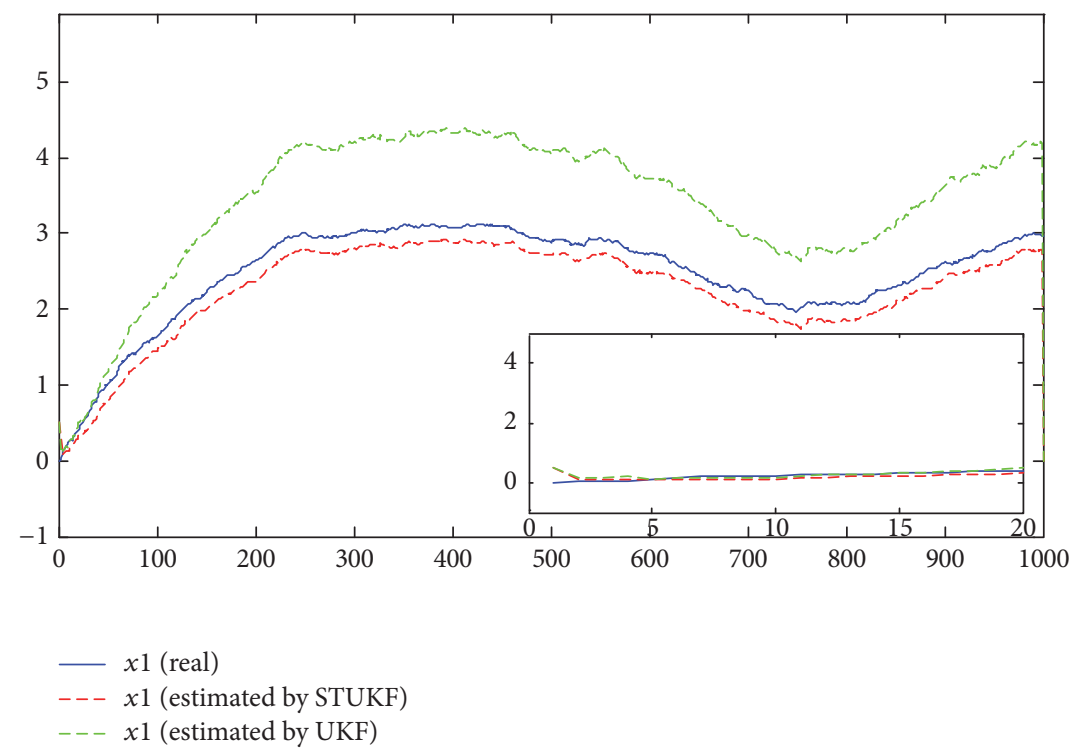

FIGURE 1: Estimation by STUKF and estimation by UKF for $x_{1, k-1}$.

The other parameters in the system are chosen as

$$
\begin{aligned}
T_{r} & =0.4660, \\
K & =259.5, \\
\mu & =85.89, \\
M & =0.068, \\
L_{r} & =0.0699, \\
J & =0.0586, \\
p & =1, \\
h & =0.001 .
\end{aligned}
$$

\section{Choose}

$$
S=\left[\begin{array}{cccccccccc}
-1 & 0 & 0 & 0 & 0 & -1 & 0 & 1 & 0 & 0 \\
1 & -1 & 0 & 1 & 0 & 0 & -1 & 0 & 0 & 0 \\
0 & -1 & 0 & 1 & 0 & 0 & -1 & 0 & 1 & 0 \\
0 & 0 & -1 & -1 & -1 & -1 & -1 & 0 & 1 & 0 \\
0 & -1 & 0 & 0 & -1 & -1 & -1 & 0 & 0 & 1
\end{array}\right]
$$

Using (2), it is obtained that

$$
T=\left[\begin{array}{ccccc}
0.5 & 0 & -0.5 & -0.5 & 0 \\
0.5 & 0.5 & 0 & 0.5 & 0 \\
0 & 0 & 0.5 & 0.5 & 0 \\
0.5 & 0.5 & -0.5 & -0.5 & -1 \\
0.5 & 0 & -0.5 & -0.5 & -1
\end{array}\right]
$$

TABLE 1: Computation times of the UKF and STUKF per run for the simulation cases.

\begin{tabular}{lc}
\hline Algorithms & Simulation time (s) \\
\hline UKF & 0.03164 \\
STUKF & 0.05385 \\
\hline
\end{tabular}

TABLE 2: RMSE of the estimation in UKF and STUKF.

\begin{tabular}{lccccc}
\hline \multirow{2}{*}{ Algorithm } & \multicolumn{5}{c}{ Error } \\
& $x_{1, k-1}$ & $x_{2, k-1}$ & $x_{3, k-1}$ & $x_{4, k-1}$ & $x_{5, k-1}$ \\
\hline UKF & 1.1084 & 0.7880 & 0.1266 & 0.0538 & 0.0537 \\
STUKF & 0.1732 & 0.1827 & 0.0644 & 0.0382 & 0.0367 \\
\hline
\end{tabular}

$$
N=\left[\begin{array}{ccccc}
0.5 & 0 & 0.5 & 0.5 & 0 \\
0 & 0 & 0.5 & -0.5 & 0 \\
0 & 0 & 0.5 & -0.5 & 0 \\
-0.5 & -0.5 & 0.5 & 1.5 & 0 \\
-0.5 & 0 & 0.5 & 0 & 1
\end{array}\right]
$$

It can be verified that $T$ has full rank.

The previous simulations were carried out with MATLAB programs on a Pentium T4400 $2.2 \mathrm{GHz}$ and $2 \mathrm{~GB}$ memory PC. Table 1 shows the average computation times of the UKF and STUKF for each run of 100 Monte Carlo simulations for the simulation case.

Using the STUKF, the state estimation results of $x_{1, k-1}, x_{2, k-1}, x_{3, k-1}, x_{4, k-1}$, and $x_{5, k-1}$ obtained by STUKF comparison with the one obtained by UKF are separately shown in Figures 1-5.

The Root Mean Square Error (RMSE) of the estimation for the proposed sampled-data descriptor system is shown in Table 2. 


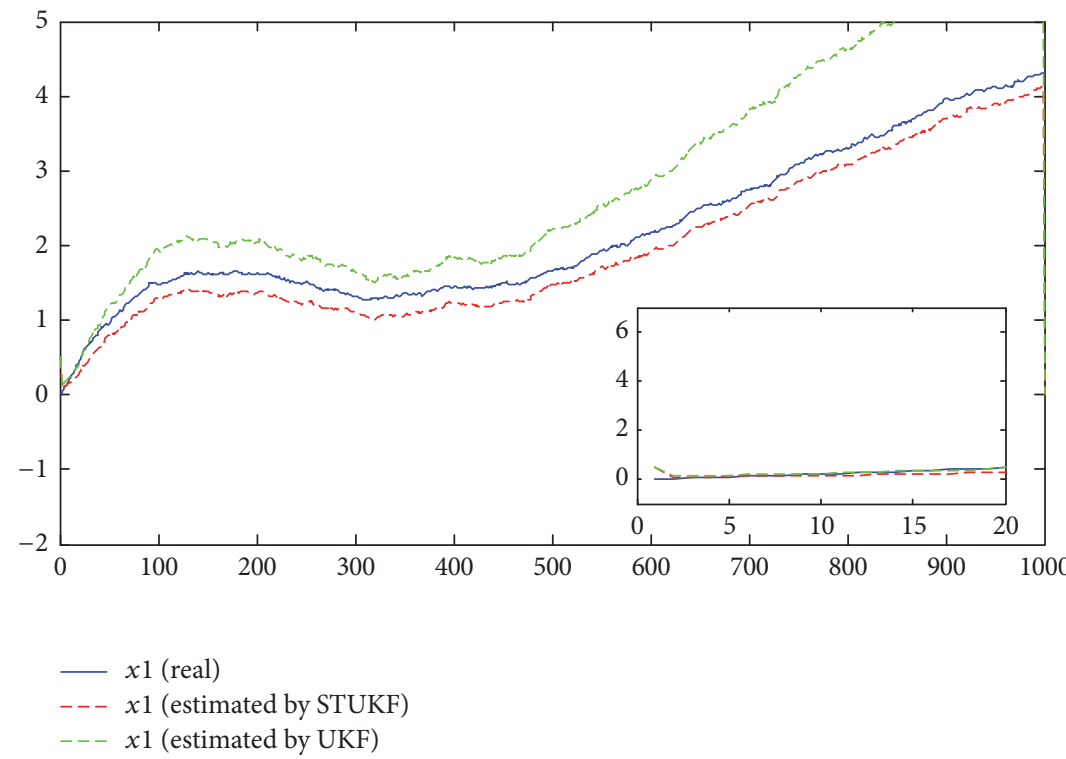

FIGURE 2: Estimation by STUKF and estimation by UKF for $x_{2, k-1}$.

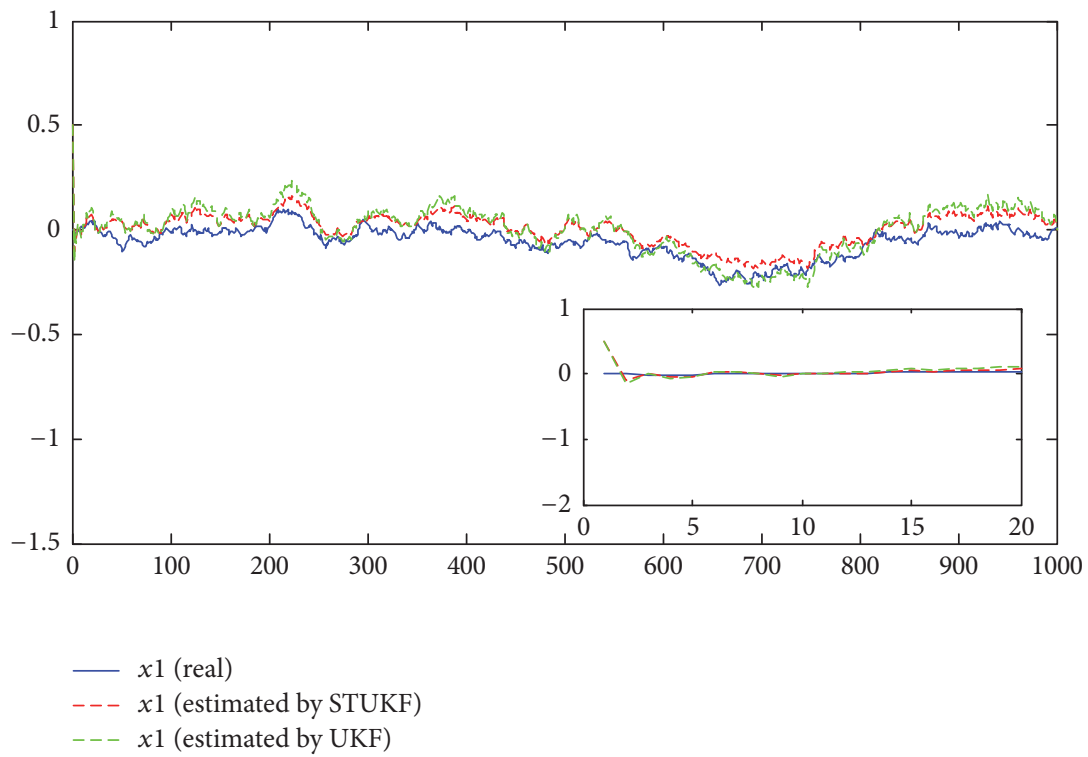

FIgURE 3: Estimation by STUKF and estimation by UKF for $x_{3, k-1}$.

Figures 1-5 show the performance of the STUKF algorithm and the UKF algorithm for the transformed nonlinear system (12). As expected, the STUKF method can achieve more accurate estimation results.

It can be seen that, for the state estimation of the transformed nonsingular system (12), the UKF method is infeasible because of the increasing noise in the state of (12). The estimation curves shown in Figure 1 to Figure 5 obtained by UKF indicate that the standard UKF algorithm cannot estimate the state of the proposed system accurately.

On the contrary, as a suboptimal fading factor $\lambda_{k}$ introduced into the predicted covariance, $K_{k}$ can adjust the influence in corresponding covariance caused by the increasing noise. The estimation curves by the STUKF algorithm of system (12) approximate to the state of real system (1). From Figures $1-5$, it can be seen that the fluctuations in the error covariance are high at the very beginning of the simulation but soon become more steady as the simulation proceeds. The simulation curves of the state and estimation by STUKF similarly coincide with each other, which means the error covariance is sufficiently small in estimation of $x_{1, k-1}$ to $x_{5, k-1}$. The RMSE in UKF and STUKF further verify this result. By the STUKF algorithm, the RMSE of the state vectors for the proposed systems are highly decreased.

Obviously, the STUKF algorithm can effectively estimate the state of the proposed sampled-data descriptor nonlinear system (1). 


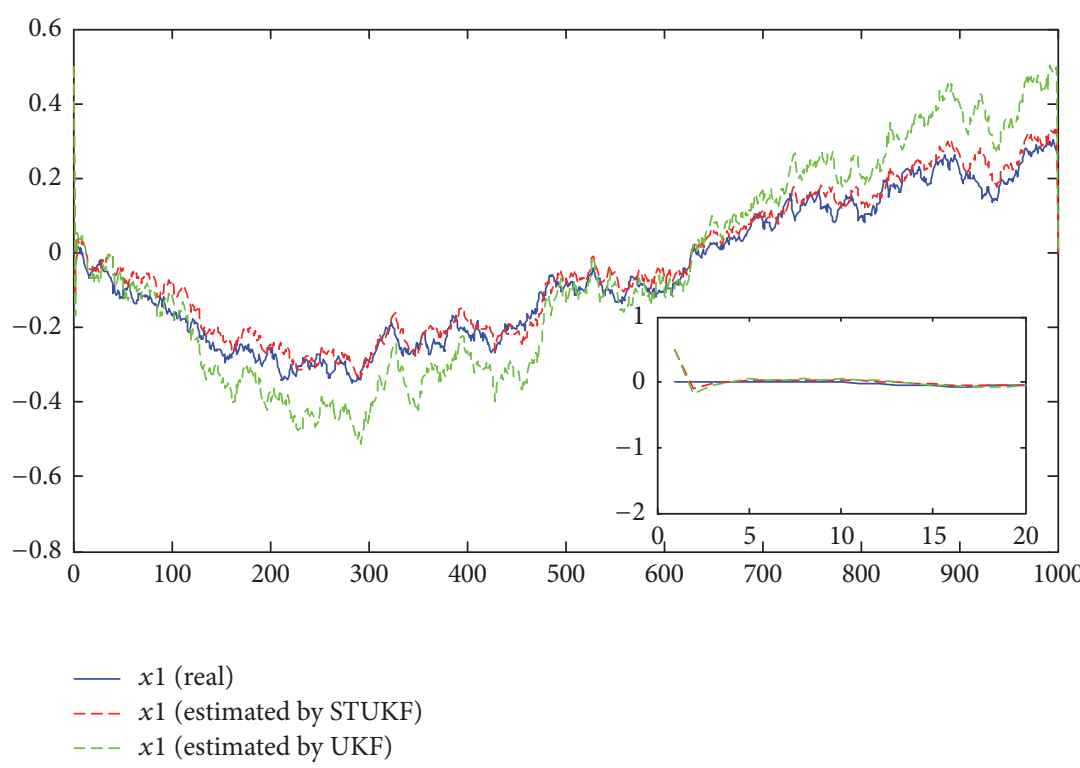

FIGURE 4: Estimation by STUKF and estimation by UKF for $x_{4, k-1}$.

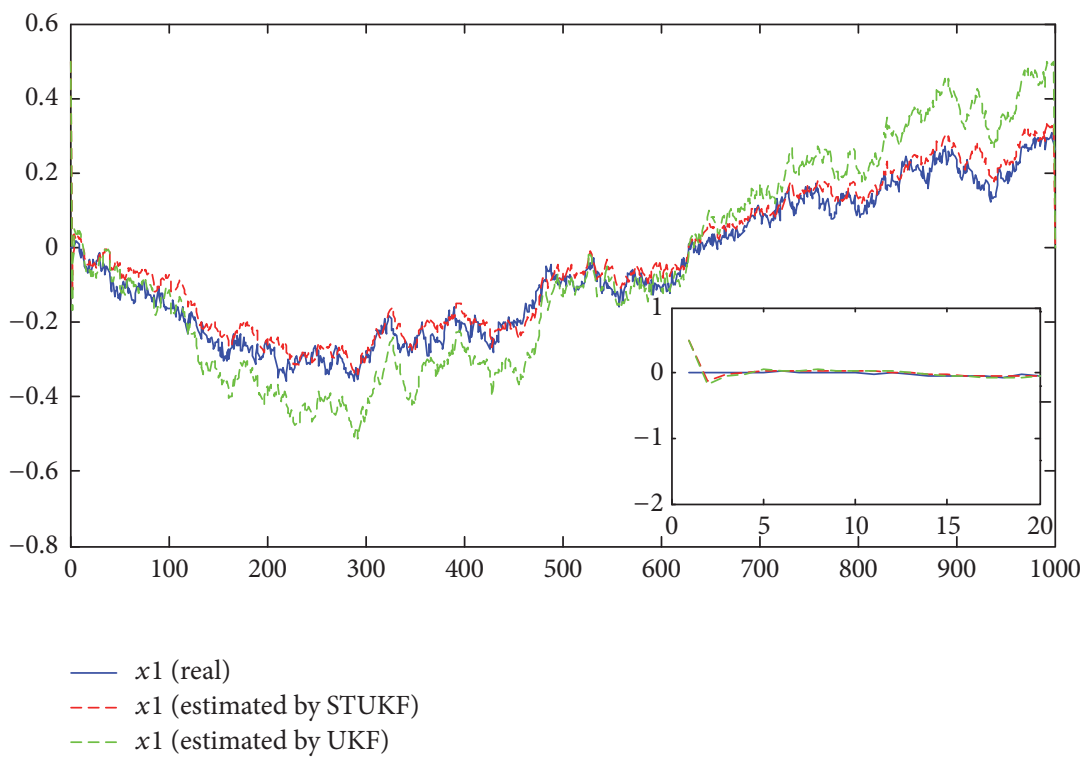

FIGURE 5: Estimation by STUKF and estimation by UKF for $x_{5, k-1}$.

\section{Conclusion}

The state estimation of the sampled-data descriptor systems by the Kalman filter method is introduced in this paper, and a novel approach of the modified strong tracking Kalman filter (STUKF) is proposed for the discretized nonsingular system. As parameters are introduced for transforming the descriptor system into a nonsingular one, there exists an increasing noise in the state of the system. To solve this problem, a defined suboptimal fading factor based on the orthogonality principle of the innovation vectors in the framework of the UKF is introduced into the prediction covariance to adjust the Kalman gain matrix. Then, the simulation example is used to demonstrate the effectiveness of the design procedure which can be implemented.

\section{Conflicts of Interest}

The authors declare that there are no conflicts of interest regarding the publication of this paper.

\section{References}

[1] Y. Uetake, "Adaptive observer for continuous descriptor systems," Institute of Electrical and Electronics Engineers. Transactions on Automatic Control, vol. 39, no. 10, pp. 2095-2100, 1994. 
[2] L. Dai, "Singular control systems," in Lecture Notes in Control and Information Sciences, vol. 118, Springer, 1989.

[3] G.-R. Duan, Analysis and Design of Descriptor Linear Systems, Springer, New York, NY, USA, 2010.

[4] B. L. Stevens and F. L. Lewis, Aircraft Modeling, Dynamics and Control, Wiley, New York, NY, USA, 1991.

[5] B. Zhang, "Parametric eigenstructure assignment by state feedback in descriptor systems," IET Control Theory \& Applications, vol. 2, no. 4, pp. 303-309, 2008.

[6] Z. Gao and S. X. Ding, "Fault estimation and fault-tolerant control for descriptor systems via proportional, multipleintegral and derivative observer design," IET Control Theory \& Applications, vol. 1, no. 5, pp. 1208-1218, 2007.

[7] M. Darouach and M. Boutayeb, "Design of observers for descriptor systems," Institute of Electrical and Electronics Engineers. Transactions on Automatic Control, vol. 40, no. 7, pp. 13231327, 1995.

[8] D. Koenig and S. d. Mammar, "Design of proportional-integral observer for unknown input descriptor systems," Institute of Electrical and Electronics Engineers. Transactions on Automatic Control, vol. 47, no. 12, pp. 2057-2062, 2002.

[9] D. Koenig, "Observer design for unknown input nonlinear descriptor systems via convex optimization," Institute of Electrical and Electronics Engineers. Transactions on Automatic Control, vol. 51, no. 6, pp. 1047-1052, 2006.

[10] R. Nikoukhah, A deterministic and stochastic theory for twopoint boundary-value descriptor systems [Ph.D. Thesis], Electrical Engineering and Computer Science Department, Massachusetts Institute of Technology, Cambridge, Mass, USA, 1988.

[11] R. Nikoukhah, B. C. Levy, and A. S. Willsky, "Generalized Riccati equations for two-point boundary-value descriptor systems," in Proceedings of the 26th IEEE Conference on Decision and Control, vol. 34, pp. 1140-1141, Laboratory for Information and Decision Systems, Massachusetts Institute of Technology, Los Angeles, Calif, USA, December 1987.

[12] X.-M. Wang and P. Bernhard, "Filtrage et lissage des systkmes discrets," Tech. Rep. 1083, Institut National de Recherche en Informatique et Automatique, Rocquencourt, France, 1989.

[13] V. Mehrmann, "Existence, uniqueness, and stability of solutions to singular linear quadratic optimal control problems," Linear Algebra and Its Applications, vol. 121, pp. 291-331, 1989.

[14] M. Darouach, M. Zasadzinski, and D. Mehdi, "State estimation of stochastic singular linear systems," International Journal of Systems Science. Principles and Applications of Systems and Integration, vol. 24, no. 2, pp. 345-354, 1993.

[15] M. Darouach, M. Zasadzinski, A. B. Onana, and S. Nowakowski, "Kalman filtering with unknown inputs via optimal state estimation of singular systems," International Journal of Systems Science, vol. 26, no. 10, pp. 2015-2028, 1995.

[16] R. Nikoukhah, A. S. Willsky, and B. C. Levy, "Kalman filtering and Riccati equations for descriptor systems," Institute of Electrical and Electronics Engineers. Transactions on Automatic Control, vol. 37, no. 9, pp. 1325-1342, 1992.

[17] R. Nikoukhah, S. L. Campbell, and F. Delebecque, "Kalman filtering for general discrete-time linear systems," Institute of Electrical and Electronics Engineers. Transactions on Automatic Control, vol. 44, no. 10, pp. 1829-1839, 1999.

[18] G. Hu, S. Gao, Y. Zhong, B. Gao, and A. Subic, "Modified strong tracking unscented Kalman filter for nonlinear state estimation with process model uncertainty," International Journal of Adaptive Control and Signal Processing, vol. 29, no. 12, pp. 1561-1577, 2015.

[19] D. H. Zhou, Y. G. Xi, and Z. J. Zhang, "A suboptimal multiple fading extended Kalman filter," Acta Automatica Sinica, vol. 17, no. 6, pp. 689-695, 1991.

[20] X. Q. Xie, D. H. Zhou, and Y. H. Jin, "Strong tracking filter based adaptive generic model control," Journal of Process Control, vol. 9, no. 4, pp. 337-350, 1999. 


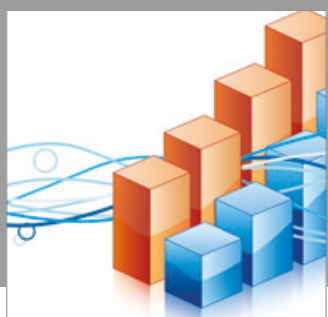

Advances in

Operations Research

vatersals

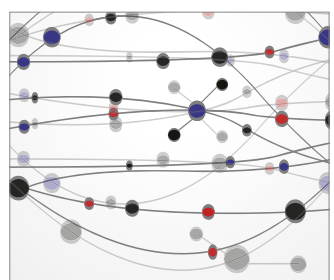

\section{The Scientific} World Journal
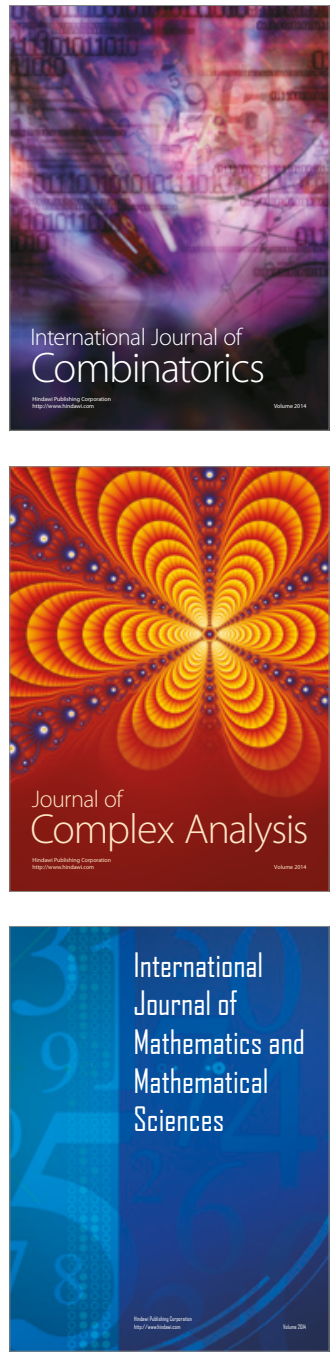
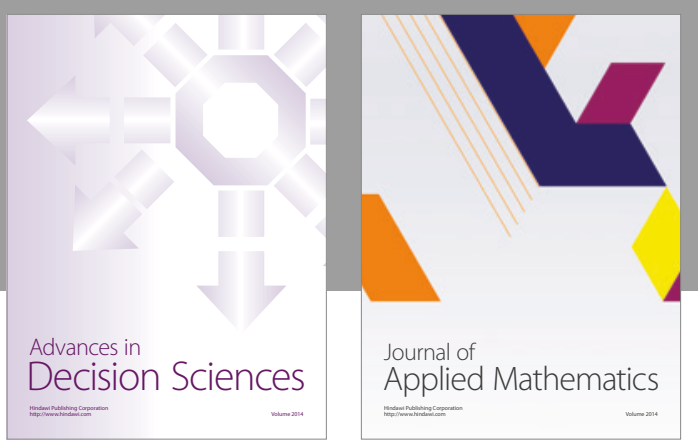

Algebra

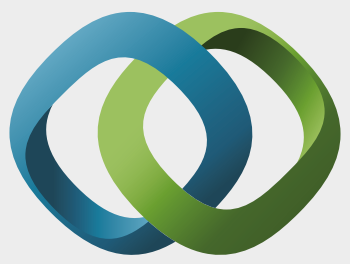

\section{Hindawi}

Submit your manuscripts at

https://www.hindawi.com
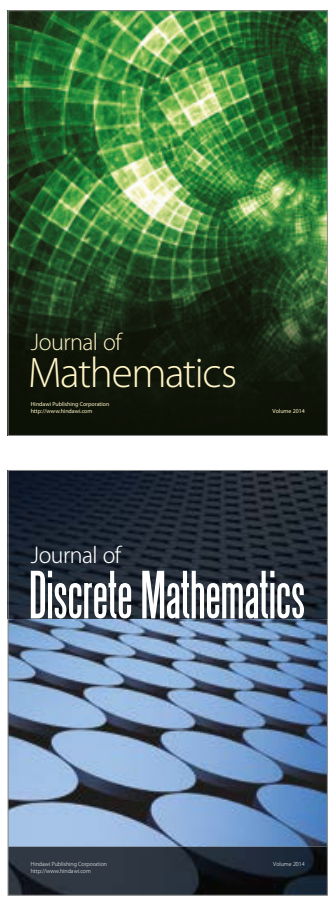

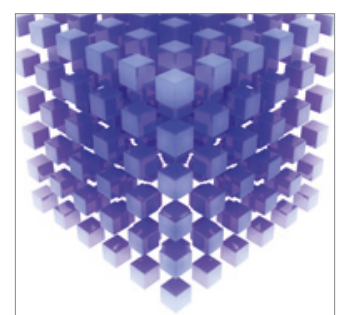

Mathematical Problems in Engineering
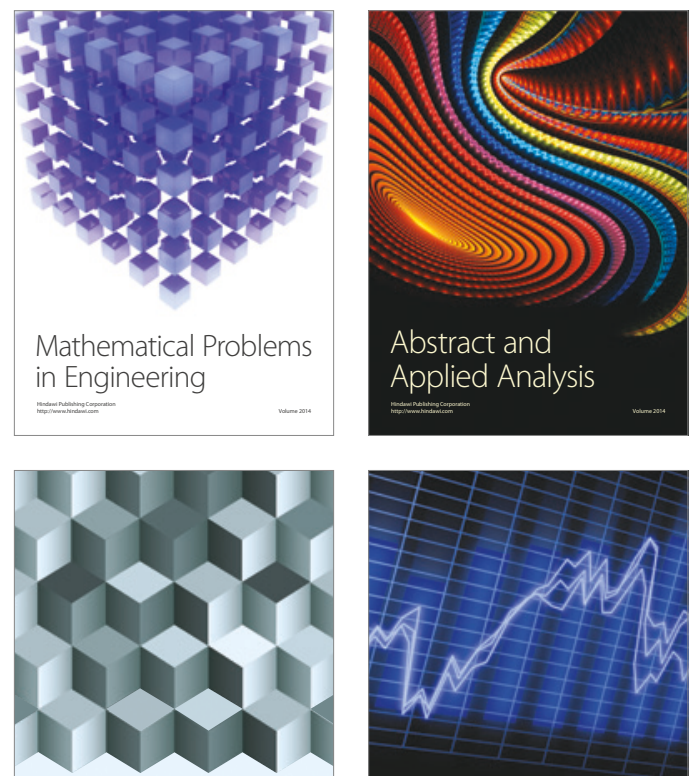

Journal of

Function Spaces

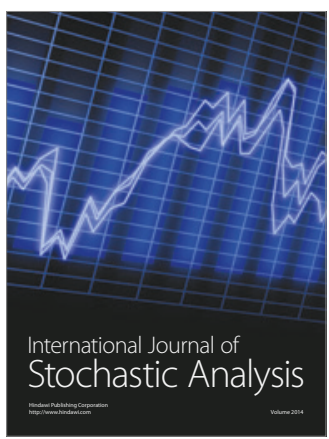

Probability and Statistics
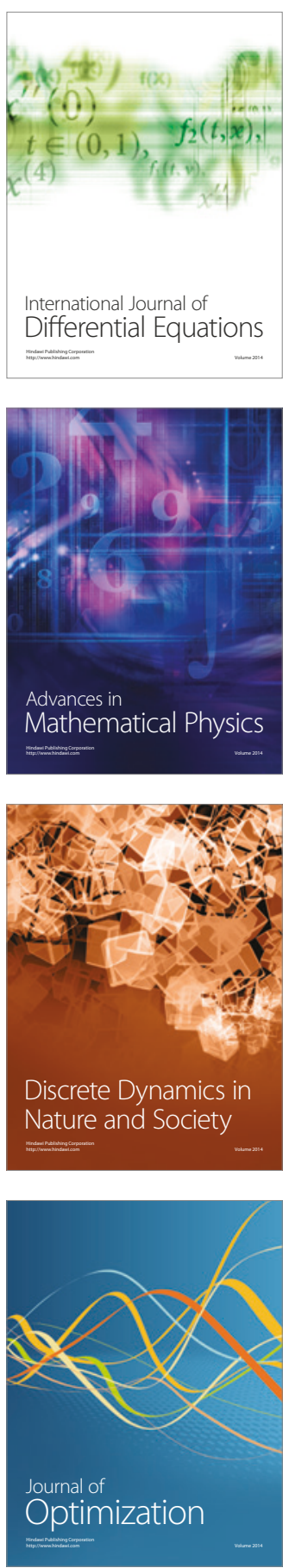